\title{
WAVE INTERACTION WITH SINGLE AND TWIN VERTICAL AND SLOPED SLOTTED WALLS
}

Noor Al Anjari, Kuwait Institute for Scientific Research, nanjari@kisr.edu.kw

Mohamad Al Khalidi, Kuwait University, mohamad.alkhaldi@ku.edu.kw

Subramaniam Neelamani, Kuwait Institute for Scientific Research, nsubram@kisr.edu.kw

\begin{abstract}
The performance of single and twin slotted walls of varying porosity and slope angles is experimentally investigated, as seen in Figure 1, in order to understand the wave-structure interaction and to asses the characteristics of wave transmission, wave reflection, and wave energy dissipation under random wave conditions. A parametric analysis was performed to understand the effects of wall porosity and slope angle, number of walls, and incoming relative wave height on the hydrodynamic characteristics of each model. The results of the study showed that reducing wall porosity from $30 \%$ to $10 \%$ decreased wave transmission up to $26.7 \%$, increased wave reflection up to $47.6 \%$, and increased wave energy dissipation by $23.7 \%$ on average. The addition of a second wall was able to reduce wave transmission by $30.2 \%$, wave reflection by $7.8 \%$, and increase wave energy dissipation by $23.3 \%$. On the other hand, decreasing slope angle lead to a reduction of $26.7 \%$ in wave transmission, $34.6 \%$ in wave reflection, and $14.7 \%$ in wave energy dissipation. Shorter waves generally lead to higher transmission, but lower reflection and energy dissipation. The results of this study showed that different wall configurations may give similar or improved results, therefore aiding in proper wall selection and reducing construction cost. It was found that using a single vertical wall of $10 \%$ porosity will transmit less wave energy than twin walls of $30 \%$ porosity and a 30-degree slope, while replacing vertical twin walls of $10 \%$ porosity with a single wall of $10 \%$ porosity and a 30-degree slope will result in higher energy dissipation, among other alternatives.
\end{abstract}

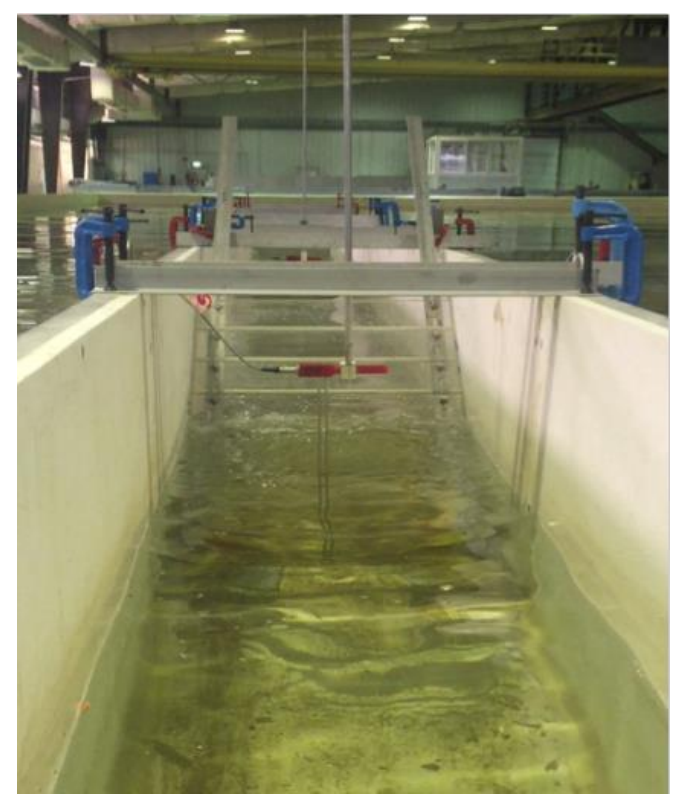

Figure 1 - Model setup in flume 